\title{
Une esthétique trash de la marginalité. Des Crasseux d'Antonine Maillet à la collection « Poésie/Rafale »(Perce-Neige)
}

\author{
Isabelle Kirouac Massicotte \\ Université de Toronto
}

Il n'est pas aisé de parler de relève en littérature acadienne, soit parce qu'on nie son existence ou encore parce qu'on peine à en discerner les contours. Dans un article intitulé «Les poètes acadiens de la relève », paru en 2005, Raoul Boudreau met le doigt sur le cœur du problème de la relève en poésie acadienne :

Il y a maintenant une bonne trentaine d'années depuis que la poésie acadienne moderne s'est manifestée au cours des années 1970. Il va sans dire que la poésie acadienne s'est beaucoup transformée au cours de ces années et que le contexte actuel de production poétique est très différent de ce qu'il était en 1970. Le passage du temps amène certains commentateurs à établir des comparaisons et certains d'entre eux à affirmer que ce qui s'écrit aujourd'hui n'est pas à la hauteur de ce qui 
ISABELLE KIROUAC MASSICOTTE, « Une esthétique trash de la marginalité. Des Crasseux d'Antonine Maillet à la collection "Poésie/Rafale " (Perce-Neige) 》

s'écrivait il y a trente ans. On entend de part et d'autre, non seulement que la qualité de la poésie acadienne est en baisse, mais aussi qu'il n’y a pas de relève. (En ligne)

Boudreau explique ensuite cette frilosité d'un certain pan de la critique à l'endroit de la production du début des années 2000; il souligne que

[1]a poésie acadienne s'est éloignée des revendications politiques et identitaires et est entrée dans une phase plus spécifiquement littéraire qui n'entraîne pas les mêmes enthousiasmes et les mêmes émotions que la fièvre des origines. Si elle y perd en charge émotive, elle y gagne en autonomie et se trouve plus libre d'explorer une gamme plus vaste d'expériences littéraires. S'il est vrai de dire qu'aucun recueil aujourd'hui ne suscite l'émotion d'un Cri de terre ou d'un Mourir à Scoudouc, il est injuste de faire une telle comparaison, car la réception enthousiaste de ces recueils tenait autant à la conjecture politique qu'aux qualités intrinsèques des recueils. (2005, en ligne)

Après avoir procédé à l'analyse des recueils de cinq «jeunes poètes » (rappelons ici que l'article date de 2005), Paul Bossé, Éric Cormier, Marie-Claire Dugas, Jean-Philippe Raîche et Christian Roy, le chercheur en vient à la conclusion que la production poétique acadienne récente se démarque par son « ouverture à des thèmes qui ne sont pas confinés à l'Acadie et qui touchent à l'expérience fondamentale de la poésie » (Boudreau, 2005, en ligne). Pour Boudreau, il importe d'appréhender les recueils publiés au tournant du XXI siècle dans leur contexte, qui n'est plus celui de la prise de parole et des débuts de l'institutionnalisation littéraire des années 1970; selon lui, cette production récente se distinguerait par ses thématiques et son traitement poétique aux penchants universels. Le problème de la relève se situerait donc surtout du côté de la réception des œuvres récentes, souvent réduites à leur pauvreté littéraire.

À l'exception de Marie-Claire Dugas, tous les poètes étudiés par Boudreau sont apparentés à l'école Aberdeen, cette tendance littéraire en Acadie identifiée pour la première fois en 2001 par la regrettée Isabelle Cossette et Manon Laparra dans leur article 
«Les voix nocturnes : modes de représentations de la cité dans la poésie acadienne contemporaine ». Il est intéressant de relever que Boudreau n’a pas jugé bon de parler de l'école Aberdeen, et ce même si son article porte précisément sur la question de la relève, ce qui vient confirmer les dires de Pénélope Cormier, qui affirme qu' « il est frappant de constater à quel point l'école Aberdeen n'occupe pas une très grande place dans le discours sur la littérature acadienne »(2012, p. 184). Parmi les chercheuses à s'être intéressées à la question, Cossette et Laparra font figures de pionnières et ont l'immense mérite d'être les premières à avoir esquissé les traits de cette «deuxième vague de poètes modernes en Acadie » (Cormier, 2012, p. 182) (on pourrait aussi parler d'une troisième génération, puisque la première serait uniquement constituée de l'écrivaine Antonine Maillet [McLaughlin, 2001, p. 136]). L'école Aberdeen réunit «de jeunes écrivains de vingt à trente ans environ [au moment de rédiger l'article en 2001] et ayant publié un ou plusieurs recueils depuis $1990 »\left(\right.$ Cossette et Laparra, 2001, p. 145). La poésie des Marc Arseneau ${ }^{1}$, Christian Brun ${ }^{2}$, Fredric Gary Comeau ${ }^{3}$, Marc Poirier $^{4}$, Éric Cormier ${ }^{5}$, Mario Leblanc ${ }^{6}$, Cindy Morais ${ }^{7}$, Matthieu Gallant ${ }^{8}$ et Christian Roy $^{9}$ « revendique de fait une certaine exiguïté, dans le sens de la marginalité »(Cossette et Laparra, 2001, p. 146). Mais cette exiguïté, cette marginalité n'est pas celle de la société acadienne par rapport à l'ensemble

${ }^{1}$ À l'antenne des oracles, Moncton, Perce-Neige, 1992; L'éveil de Lodela, Moncton, Perce-Neige, 1998.

${ }^{2}$ Tremplin, Moncton, Perce-Neige, 1996; Hucher parmi les bombardes, Moncton, Perce-Neige, 1998.

${ }^{3}$ Ravages, Moncton, Perce-Neige, 1994; Trajets, Moncton, Perce-Neige, 1996; Routes, Moncton, PerceNeige, 1997.

${ }^{4}$ Avant que tout' disparaisse, Moncton, Perce-Neige, 1993.

${ }^{5}$ À vif tel un circoncis, Moncton, Perce-Neige, 1997; Le flirt de l'anarchiste, Moncton, Perce-Neige, 2000.

${ }^{6}$ Taches de naissance, Moncton, Perce-Neige, 1999.

${ }^{7}$ Zizanie, Moncton, Perce-Neige, 1999.

8 Transe migration, Moncton, Perce-Neige, 2000.

${ }_{9}^{9}$ Pile ou face à la vitesse de la lumière, Moncton, Perce-Neige, 1999; Infarctus parmi les piétons, Moncton, Perce-Neige, 2000. 
ISABELLE KIROUAC MASSICOTTE, «Une esthétique trash de la marginalité. Des Crasseux d'Antonine Maillet à la collection “Poésie/Rafale ” (Perce-Neige) 》

de la société, comme l'a relevé avec justesse Pénélope Cormier $(2012,1999)$; on la trouve plutôt dans différentes représentations de la marginalité sociale, dans une «utilisation littéraire de notions telles que les drogues, le sexe, la violence ou le vampirisme, notions [...] qui découlent directement d'une vision de l'underground propre à ce groupe d'écrivains »(Cossette et Laparra, 2001, p. 146). Contrairement à Boudreau, Cossette et Laparra considèrent que la «poésie acadienne contemporaine ne se préoccupe pas d'une quelconque universalité littéraire » (2001, p. 146); la production des décennies 1990 et 2000 se distinguerait donc par son délaissement de l'acadianité - il y a ici consensus - au profit d'une esthétisation de différents types de marges.

À la suite de Cossette et de Laparra, Pénélope Cormier a elle aussi grandement contribué à définir le mouvement de l'école Aberdeen, notamment en lui ajoutant le nom de quelques de poètes: Daniel Omer LeBlanc ${ }^{10}$, Stéphanie Morris ${ }^{11}$ et Sarah Marylou Brideau $^{12}$. Le travail de Cormier se distingue en ce qu'il aborde de front la question de la relève poétique acadienne et qu'il dresse un portrait institutionnel fort révélateur. La chercheuse indique que la « littérature acadienne des années 1990 est bien la proie d'une restructuration fondamentale de la logique de fonctionnement du champ littéraire » (Cormier, 2012, p. 186). Pour Cormier, la « restructuration du champ ne passe [...] plus tant par le conflit vertical entre l'Ancien et le Moderne [elle fait ici référence au conflit intergénérationnel opposant Antonine Maillet aux poètes des années 1970] que par une diversification des pratiques "horizontales" »(2012, p. 186). Par pratiques horizontales, la chercheure fait référence au conflit à l'intérieur d'une même génération, plus

\footnotetext{
${ }^{10}$ Les ailes de soi, Moncton, Perce-Neige, 2000.

${ }^{11}$ Le risque des rêves, Moncton, Perce-Neige, 2002.

12 Romanichelle, Moncton, Perce-Neige, 2002; Rues étrangères, Moncton, Perce-Neige, 2004.
} 
particulièrement celui opposant Gérald Leblanc à Herménégilde Chiasson; dès lors, l'idée de relève se voit complexifiée, car elle ne dépend plus d'une rupture franche avec la génération précédente pour advenir. En illustrant son propos à partir d'une citation de Claude Beausoleil, Cormier relève avec justesse que le fait de trouver une façon de dire les choses autrement devient le principal enjeu des nouvelles formes poétiques; la relève en poésie acadienne est « $[\mathrm{u}]$ rbaine, mais autrement. Intime, mais autrement. Engagée, mais autrement. C'est dans cet "autrement" que l'on trouve des pistes pour entrer dans le corpus de la poésie récente » (Beausoleil, 1999, p. 111).

Ces remarques sur la relève et sur sa façon de dire autrement permettent d'appréhender une quatrième générations d'écrivaines et d'écrivains acadiens, qui regroupe les Jonathan Roy ${ }^{13}$, Gabriel Robichaud ${ }^{14}$, Sébastien Bérubé ${ }^{15}$, Joannie Thomas ${ }^{16}$ et Monica Bolduc $^{17}$. À l'exception de Roy et de Robichaud, tous ces jeunes poètes sont publiés dans la collection « Poésie/Rafale », chez Perce-Neige, à Moncton, qui «joue le rôle qu'occupait la maison d'édition Perce-Neige à ses débuts : publier un premier recueil d'un nouvel auteur » (Doyon-Gosselin, 2016, en ligne). Mais la parenté esthétique des œuvres de «Poésie/Rafale » relevée par Benoit Doyon-Gosselin s'applique tout autant à la poésie de Roy et de Robichaud : « les textes de cette collection proposent une poésie coup-de-poing, plus sale, plus trash même»(2016, en ligne). Loin d'être en rupture complète avec la génération qui l'a précédée (celle des poètes d'Aberdeen), cette quatrième génération

${ }^{13}$ Apprendre à tomber, Moncton, Perce-Neige, 2012.

${ }^{14}$ La promenade des ignorés, Moncton, Perce-Neige, 2011; Les anodins, Moncton, Perce-Neige, 2014; Acadie Road, Moncton, Perce-Neige, 2018.

${ }_{15}$ Sous la boucane du moulin, Moncton, Perce-Neige, 2015; Là où les chemins de terre finissent, Moncton, Perce-Neige, 2017.

${ }^{16}$ Quatre pattes Catherine, Moncton, Perce-Neige, 2016.

${ }^{17}$ Dead end, Moncton, Perce-Neige, 2017. 
ISABELLE KIROUAC MASSICOTTE, « Une esthétique trash de la marginalité. Des Crasseux d'Antonine Maillet à la collection “Poésie/Rafale ” (Perce-Neige) »

s'applique elle aussi à dire la marginalité, mais autrement, d'autant plus que les vers de Roy et de Bérubé donnent à lire une certaine « revanche des régions » (Doyon-Gosselin, 2017, en ligne) (la poésie de l'école Aberdeen est profondément urbaine, rappelons-le). À mon sens, cette question de dire la marginalité autrement traverse l'ensemble du corpus acadien. Dans cette étude, je souhaite plus particulièrement m'attarder à ce que j'appelle l'esthétique trash, qui illustre le fait marginal dans les différents moments de la littérature acadienne. J'argue que les poètes de l'école Aberdeen - et ceux de la collection «Poésie/Rafale »poursuivent, mais autrement, l'esthétique trash acadienne qui est également à l'œuvre dans la pièce de théâtre Les Crasseux (1973 [1968]) d'Antonine Maillet et les recueils fondateurs de la modernité acadienne, Cri de terre (2012 [1972]) de Raymond Guy LeBlanc et Acadie Rock (1994 [1973]) de Guy Arsenault.

À partir de l'esthétique trash que je conceptualise et en prenant pour principaux exemples les œuvres de l'école Aberdeen et celles de la collection «Poésie/Rafale », je me propose d'aborder l'évolution de cette esthétique. Avec cette étude, je souhaite notamment démontrer que le fait d'appréhender la littérature acadienne d'après la lorgnette du trash littéraire constitue l'une des façons de réévaluer notre façon de catégoriser les œuvres et de rattacher la production récente au corpus acadien consacré.

\section{L'esthétique trash}

D'entrée de jeu, il me faut expliciter ce que j'entends par esthétique trash. Une esthétique trash est difficile à circonscrire; on qualifie régulièrement des œuvres de trash sans toutefois intellectualiser et théoriser la notion. En outre, dans le domaine des arts, le trash est souvent abordé comme étant opposé aux œuvres classiques, comme produit artistique de piètre qualité destiné à la«consumer society» (Keller Simon, 1999, p. 7; voir aussi 
Brottman, 2005, au sujet de la culture trash et du mauvais goût dans tous ses états). Or il ne s'agit pas là de l'acception que je donne au trash, que j'étudie plutôt en tant qu'esthétique de la marginalité. Le trash est évoqué le plus souvent trop rapidement et dans sa version caricaturale : il serait nécessairement gore, violent, cru et vulgaire. Je rejette cet emploi rapide et facile du trash parce qu'il ne permet pas d'appréhender la richesse du concept, qui a d'autres racines, notamment en philosophie.

Le philosophe Greg Kennedy propose une ontologie du trash dans son essai An Onthology of Trash (2007). Son étude vise à déterminer «how and why beings have become disposable »(Kennedy, 2007, p. 13). Pour Kennedy, il existe une relation entre la production de déchets et les inégalités sociales : «any society, such as our own, that generates gross amounts of waste must have correspondingly gross inadequacies » (2007, p. 3-4). À l'ère du jetable, les objets et les individus sont tributaires de leur « utilité », de la valeur qu'on leur attribue. Si l'utilité d'une personne n'est pas immédiatement identifiable par la société dominante, celle-ci se retrouve disqualifiée, reléguée aux marges, traduisant un rapport inégal au pouvoir. Ainsi, la dévaluation ne s'applique pas seulement aux choses, mais également aux êtres : «Since values are our investment into things, their substraction marks our divestment from or indifference to things »(Kennedy, 2007, p. 5). Le philosophe souligne que « uncaring as the mode of a possible being-in-the-world is an existential mode of violence that negates the being of beings. This ontological violence is the progenitor of trash» (Kennedy, 2007, p. 144). Cette dévaluation absolue engendre le trash, qui tend vers la disparition - elle se situerait dans sa suite logique (Kennedy, 2007, p. 52). Cependant, « the real significance of trash is affirmative, not negative » (Kennedy, 2007). Une fois ses caractéristiques les plus évidentes dépassées, le trash représente une importante opportunité 
ISABELLE KIROUAC MASSICOTTE, «Une esthétique trash de la marginalité. Des Crasseux d'Antonine Maillet à la collection “Poésie/Rafale ” (Perce-Neige) 》

d'édification et de transformation (Kennedy, 2007, p. 206) se traduisant par l'avènement d'un monde plus vrai : «the truth of trash indicates our inauthenticity (Kennedy, 2007, p. 184).

À l'instar de Kennedy, Kenneth Harrow, auteur de l'essai Trash. African Cinema From Below (2013), réfléchit à la trajectoire du trash, pensée en termes d'absence et de manque de valeur: "the descent is here only because of the belief that the depiction of it will serve to enable a change, an ascent, to become possible » (Harrow, 2013, ePub). Harrow réfléchit à la signification du trash :

trash, above all, applies to people who have been dismissed from the community, marginalized and forgotten. [...] Trash encompasses the turning of that reduced status into the basis for revolt, change, and the turning away from regimes that produce definitions of trash to newly formulated regimes that force us to reconsider the criteria of assigning value (Harrow, 2013, ePub).

Harrow insiste sur la fonction du trash: «to show the face of oppression and worthlessness »(Harrow, 2013, ePub) de celles et ceux considérés comme les déchets humains, les personnes marginalisées : les gens du «bas ». Selon Harrow, il existe deux façons pratiquement opposées de montrer et de dire le trash; «trash in the spectacular sense of trash [...]. That is trash that flaunts its extravaganza [...] But the déchets humains [...] belong to another domain of representation, ethical in its demand and representational »(Harrow, 2013, ePub). Le trash peut donc être obscène - comme c'est le cas du discours pornographique, qui montre ce qui ne doit pas être vu - mais dans sa signification la plus littérale, il peut également être signifié par une précarité de l'écriture, plus près de sa tendance à la disparition. Le trash est donc intrinsèquement ambigu et de nature changeante : «it is always in a state of change, losing and gaining value, shifting 
regimes, always in transition » (Harrow, 2013, ePub). Il n'est donc pas possible - ni souhaitable - de fournir une définition toute faite de ce qu'est le trash.

Harrow soulève en outre la notion de trash global, «that attests to the nature of a system generated by unequal relations of power, exacerbated under colonialism and neoliberal globalization » (Harrow, 2013, ePub). Et j'ajouterais le patriarcat, un autre vecteur de rapports inégaux au pouvoir. Le trash met en évidence des problématiques liées à la marginalité sous toutes ses formes, qu'elle soit raciale, de classe ou de genre; Harrow présente la figure de la trashy woman en ces mots : «Trashy women are dangerous because they are fallen women whose eroticism threatens male power and control». La femme trash, «treated like trash and called trashy»(Harrow, 2013, ePub), trouve son pouvoir dans son érotisme.

Pour appuyer son étude du trash, Harrow a recours à certains concepts du philosophe français Jacques Rancière. Bien que Rancière ne parle pas explicitement du trash, sa pensée sur le partage du sensible et le dissensus résonne avec la question du trash. Le penseur réfléchit le partage du sensible en ces termes :

C'est un découpage des temps et des espaces, du visible et de l'invisible, de la parole et du bruit qui définit à la fois le lieu et l'enjeu de la politique comme forme d'expérience. La politique porte sur ce qu'on voit et ce qu'on peut en dire, sur qui a la compétence pour voir et la qualité pour dire, sur les propriétés des espaces et les possibles du temps. (2000, ePub)

Mais tous ne sont pas égaux devant ce partage du sensible, car il

fixe [...] en même temps un commun partagé et des parts exclusives. [...] Le partage du sensible fait voir qui peut avoir part au commun en fonction de ce qu'il fait, du temps et de l'espace dans lesquels cette activité s'exerce. Avoir telle ou telle occupation définit ainsi des compétences ou des incompétences au commun. Cela définit le fait d'être ou non visible dans un espace commun, doué d'une parole commune, etc. (2000, ePub) 
ISABELLE KIROUAC MASSICOTTE, «Une esthétique trash de la marginalité. Des Crasseux d'Antonine Maillet à la collection “Poésie/Rafale ” (Perce-Neige) 》

Ce système de sens de l'ordre dominant est producteur de la «garbagization » dont parle Cecily F. Brown, terme qu'elle a créé pour nommer une dynamique sociale et sa rhétorique qui utilise les déchets comme métaphore pour certains individus, ainsi définis comme des êtres jetables, des êtres en trop. Pour Brown, cette dynamique sert à renforcer et à réitérer les hiérarchies, qu'elles soient de classe, de genre ou de race. Selon Rancière, la politique consiste en la reconfiguration du partage du sensible qui détermine le commun d'une communauté en y introduisant des objets nouveaux, en rendant visible ce qui ne l'était pas et en faisant entendre les locuteurs qui étaient réduits au silence. L'esthétique trash crée un dissensus, car elle montre ce que l'on ne veut ni voir, ni entendre, ni même sentir dans l'ordre dominant; l'esthétique trash vient troubler et déstabiliser les systèmes culturels. L'une des fonctions du trash est de reconfigurer le partage du sensible et d'opérer une certaine révolution de la représentation.

L'esthétique trash, telle qu'elle est esquissée par Kennedy et Harrow, peut également être apparentée à la théorie de la minorisation formulée par François Paré, notamment dans ses essais Les littératures de l'exiguité (1992), Théories de la fragilité (1994), La distance habitée (2004) et Le fantasme d'Escanaba (2007). Paré touche du doigt le trash, mais sans le nommer ainsi, quand il mentionne l'existence dans les littératures contemporaines «d'exemples de marginalités moins tendres, dont la violence déchaînée [représente] l'envers de l'indifférence des choses » (1992, p. 20). À l'instar des «petites » littérature, le trash «s'érig[e] contre le pouvoir, contre l'arrogance de l'universel » (1992, p. 7). Emblématique de la marge, le trash est « le symbole du refus de disparaître » (1992, p. 12), la trace des communautés jetables que certaines et certains préféreraient ne pas voir et qui viennent troubler l'ordre dominant. La théorie du trash a un important potentiel 
intersectionnel (l'intersectionnalité concerne les formes de domination [genre, race, classe sociale, etc.] dans les liens qui se nouent entre elles); tous les groupes minoritaires ayant en commun une «expérience de l'écart» (1992, p. 16), un rapport inégal au pouvoir. À l'instar de Fernand Dorais, que Paré prend comme exemple d'auteur en milieu minoritaire, le trash est une esthétique où «l'espoir, très nietzschéen, surgit au terme de la plus grande déchéance » (1994, p. 29). Le trash comprend les caractéristiques de la misère, de l'oubli, de la désolation et de la destruction, mais également, du moins potentiellement, leur renversement : cette esthétique n'est pas que dysphorique, car sa crudité et sa violence peuvent déboucher sur l'indignation, la solidarité, la contestation, voire même le militantisme.

Le trash ne se limite pas à une simple définition du dictionnaire. Il demeure lié à la question des déchets et d'un statut social bas, car l'assignation de la valeur et la dévaluation sont cruciales pour comprendre la notion. Avec cette présentation de l'esthétique trash, mon objectif n'est pas d'en fournir une définition fixe, puisqu'il n'y en a pas, le trash, rappelons-le, étant toujours dans un état de changement, perdant et gagnant de la valeur, toujours en transition. En plus de sa trajectoire, l'esthétique trash peut être identifiée à partir de ses deux sens opposés; celui qui est extravagant (qui peut se traduire par un langage cru, un discours pornographique ou des images gore) et l'autre plus littéral et qui réside dans les non-dits et dans un champ sémantique du silence et de la disparition. Le trash ouvre le partage du sensible à celles et à ceux qui n'ont pas été entendus ou vus; avec ostentation à partir d'une écriture des sens et de l'abjection, ou de façon parcimonieuse, avec une écriture des détritus et des traces. L'esthétique trash, peu importe la forme qu'elle 
ISABELLE KIROUAC MASSICOTTE, «Une esthétique trash de la marginalité. Des Crasseux d'Antonine Maillet à la collection “Poésie/Rafale ” (Perce-Neige) 》

prend, est le symptôme du rapport inégal au pouvoir expérimenté par les groupes marginalisés.

\section{Une archéologie du trash dans la littérature acadienne}

D'après mes recherches, la première occurrence de l'esthétique trash en littérature acadienne remonterait à $1968^{18}$ avec la création de la pièce de théâtre Les Crasseux, signée Antonine Maillet. Précisons d'entrée de jeu que mon propos ne vise pas à présenter Maillet comme la mère du trash en Acadie ou encore à qualifier son œuvre de foncièrement trash, mais bien à souligner qu'une esthétique trash traverse Les Crasseux. Au-delà du titre qui annonce des personnages de laissés-pour-compte réduits aux signes de la saleté et de la répugnance qu'ils suscitent, l'œuvre met en jeu une véritable lutte des classes entre les gens d'En-haut - personnes respectables qui s'expriment dans un français standard et qui ont la réputation d'appartenir à « une race en santé, propre et digne » (Maillet, 1973, p. 19) - et les gens d'En-bas, les Crasseux, qui parlent un vernaculaire parsemé de jurons. La pièce est également le lieu d'une lutte pour l'espace - cela n'est pas sans rappeler le découpage des espaces qui définit le partage du sensible selon Rancière -, alors que le territoire des Crasseux, situé au pied de la voie ferrée, ne leur appartient pas au sens de la loi. Les Crasseux et leur crasse sont «a matter that is out of place », pour reprendre les mots de l'anthropologue Mary Douglas (citée dans Harrow, 2013, ePub). Les gens d'En-bas sont relégués à la marge, jugés incompétents au commun : la dynamique de «garbagization» est bien à l'œuvre, car elle opère « a sharp distinction between us [les gens d'En-haut] and them [les Crasseux] »(Brown, 2012, p. 3). Plus encore, cette «garbagization » doit être comprise comme une «social dynamic and its rhetoric, which uses garbage, waste, trash,

\footnotetext{
${ }^{18}$ L'édition utilisée dans la présente étude est celle de 1973.
} 
rubbish, etc., as a metaphor for people, so that they are understood to be disposable, redundant, and/or excess »(Brown, 2012, p. 1). Cette rhétorique a aussi pour fonction de rendre les «déchets humains » responsables de leur malheur. Cela est explicité dans les paroles de la mairesse du village d'En-Haut : «Les pauvres d'En-bas ne sont pas de vrais pauvres, Docteur, ce sont des mal élevés » (Maillet, 1973, p. 39). Devant l'expulsion qui les menace - ils seront expropriés au nom de «l'hygiène publique » (Maillet, 1973, p. 37) -, les gens d'En-bas ont pour seules armes un langage cru et l'invective - les «sacordgé! », les «batêche! » les «godêche de hell! », et les «salopes!» viennent ponctuer l'écriture mais surtout leur inventivité. Une fois expropriés de leur terre, les Crasseux s'approprient la ruse des gens d'En-haut qui les a dépouillés de leur terre : acheter un terrain qui n'appartient à personne pour la somme symbolique de $1 \$$. C'est ainsi que les Crasseux déménagent derrière la boutique du barbier, dans le village d'En-haut.

Cela est représentatif du potentiel de transformation du trash, dans sa valeur révolutionnaire. Pour Rancière, la révolution esthétique passe par des « formes d'annulation ou de renversement de l'opposition du haut et du bas »(2000, ePub). Plus encore, cette révolution est rendue possible par des énoncés littéraires qui

introduisent dans les corps collectifs imaginaires des lignes de fracture, de désincorporation [...] qui contribuent à la formation de collectifs d'énonciation qui remettent en question la distribution des rôles, des territoires et des langages - en bref, de ces sujets politiques qui remettent en cause le partage donné du sensible » (Rancière, 2000, ePub).

En infiltrant l'ordre dominant et en forçant un nouveau partage du sensible, les Crasseux se font les révélateurs de cet ordre. Tout au long de la pièce, ils mettent au jour l'hypocrisie de la «bonne » société : 
ISABELLE KIROUAC MASSICOTTE, « Une esthétique trash de la marginalité. Des Crasseux d'Antonine Maillet à la collection “Poésie/Rafale ” (Perce-Neige) 》

Michel-Archange - T'as ben dit : ce côté-citte de la bouchure. Je marche dans mon fumier, moi, pas dans le fumier des autres.

Citrouille - C'est qu'il sent moins mauvais, le fumier des autres.

Michel-Archange - Pas quand ils te le mettent dans la gueule, sacordgé! (Maillet, 1973, p. 16)

Cet échange est révélateur en ce qu'il rend visible le fumier et la violence des gens d'Enhaut, que l'on tente de dérober au regard sous l'apparat d'une langue policée et d'un discours hygiéniste. Selon Brown, «[t]hus garbage, dirt and/or waste is a reverse mirror not only of our behavior, but also of our social order » (2012, p. 7). Révéler le fumier revient à révéler le système et ses oppressions. Dans Les Crasseux, l'esthétique trash qui est mobilisée est liée à la métaphore des humains comme déchets et à son contrepoids, la révolution esthétique. Ici, le trash existe surtout dans sa forme la plus littérale, liée aux détritus.

Même si un pan de la critique littéraire acadienne a eu tendance à opposer l'œuvre de Maillet à celles de Raymond Guy LeBlanc et de Guy Arsenault, qui auraient fondé la modernité acadienne, je crois que ces productions partagent néanmoins une certaine parenté d'un point de vue esthétique. En effet, l'écriture de Cri de terre (2012 [1972]) et d'Acadie Rock (2013 [1973]) est également traversée par une esthétique trash. Du côté de Cri de terre, cette dernière oscille entre ses deux tendances; l'une, plus littérale avec l'utilisation d'un champ sémantique de l'enfouissement (implicitement lié aux déchets, contrairement au traitement plus explicite des détritus chez Maillet) et de la disparition (qui, rappelons-le, se situerait dans la suite logique du trash), et l'autre, extravagante avec l'emploi d'un langage cru. L'idée de refaire surface, cette volonté d'entrer dans la culture du visible, traverse l'ensemble du recueil, à commencer par le titre, qui donne l'impression d'une 
parole souterraine, entravée. L'image du souterrain, plutôt que d'être liée aux déchets et à leur enfouissement, rejoint celles de l'entêtement et de la résistance lorsqu'il est question, dans le poème intitulé «Silences », des vies «[c]omme fleurs prisonnières sous le béton » (LeBlanc, 2012 [1972], p. 16). À l'image du trash, tiraillé entre disparition et transformation, la menace de l'enfouissement et du silence est toujours présente : ces vies

S'en retournent à leurs racines faute de langage

Pourtant nous n'avons pas voulu

Ce silence dérisoire des vivants (LeBlanc, 2012 [1972], p. 16)

Afin de soustraire à l'oubli et à l'indifférence les « [e]nfants sans langage » (LeBlanc, 2012

[1972], p. 45) et les « [g]ens de [s]on pays dans l'absence d['eux]-mêmes » (LeBlanc, 2012

[1972], p. 45), le poète se fait leur porte-parole dans le poème « Cri de terre »:

Navire fantôme je suis remonté à la surface des fleuves

Vers la plénitude des marées humaines

Et j'ai lancé la foule aux paroles d'avenir

J'habite un cri de terre en amont des espérances

Larguées sur toutes les lèvres

Déjà mouillées aux soleils des chalutiers incandescents

Et toute parole abolit le dur mensonge

Des cavernes honteuses de notre silence (LeBlanc, 2012 [1972], p. 47)

La formule oxymorique «cri de terre» traduit bien l'enjeu d'un nouveau partage du sensible; ce qui est souterrain et qui n'appartiendrait donc pas au découpage de l'espace visible s'inscrit pourtant avec ostentation dans le découpage du bruit et de la parole. À l'indigne (ce qui n'est pas digne du partage du sensible) succède l'indignation, qui passe notamment par l'invective, comme c'était le cas dans Les Crasseux :

Je jure en anglais tous mes goddams de bâtard Et souvent les fuck it me remontent à la gorge Avec des Jesus-Christ projetés contre le windshield 
ISABELLE KIROUAC MASSICOTTE, « Une esthétique trash de la marginalité. Des Crasseux d'Antonine Maillet à la collection "Poésie/Rafale " (Perce-Neige) 》

Saignant medium-rare (LeBlanc, 2012 [1972], p. 57)

Ces vers témoignent de l'aliénation du poète, dont les sacres sont prononcés en anglais. Mais LeBlanc jure également ses «goddams de bâtard »; l'invective - forme d'agentivité parce que forme de protestation - passe donc aussi par le chiac, vernaculaire mêlant français et anglais, mais préservant une syntaxe française, propre au sud-est du NouveauBrunswick. LeBlanc innove d'un point de vue esthétique en introduisant le chiac dans la langue littéraire et en l'extirpant de son indignité. Un peu comme chez Maillet, l'esthétique trash se traduit par une réappropriation et une resignification de l'indigne (le fumier comme révélateur d'un ordre social dans Les Crasseux et la langue vulgaire et anglicisée comme véhicule de la protestation dans Cri de terre).

Avec Acadie Rock de Guy Arsenault, on revient plus évidemment au bas corporel et aux odeurs associées à l'esthétique trash. Dans son très populaire poème «Tableau de Back Yard », même le bas corporel, les odeurs, ce qui est normalement caché au regard, à l'instar de ce qui traîne dans notre cour arrière, ont droit de cité et provoquent un nouveau partage du sensible :

back yard senteur de chez nous senteur de mon père senteur de ma mère senteur de mon grand père senteur de mes sœurs, de mon frère senteur à moi senteur de chez nous comme si tou'l monde se connaissait back yard (Arsenault, 2013 [1973], p. 43-44)

En plus d'inscrire les siens dans le découpage des odeurs, pour reprendre la formulation de Rancière, Arsenault fait connaître une transformation (ce qui recoupe le travail de 
resignification repéré chez Maillet et LeBlanc) à l'odeur, élément du bas et de l'abject, matériau littéraire improbable, ici connotée positivement puisque décrite comme une senteur qui sert à dire la communauté, présentée comme unie. Dans Acadie Rock, le quotidien, le banal et l'ordinaire, les odeurs et les déchets (dans son poème «J'aime », le poète inclut des «paquets de cigarettes vides» et des «bouts de cigarette éteindus »[Arsenault, 2013 [1973], p. 68]) sont autant de manières de dire les humbles, ceux et celles qui font l'Acadie, et de leur donner le capital symbolique qui leur revient. Cela correspond au régime esthétique des arts dont parle Rancière, qui est « d'abord la ruine du système de la représentation » qui « définissait, avec les genres, les situations et les formes d'expression qui convenaient à la bassesse ou à l'élévation du sujet » (2000, ePub). En somme, c'est « la gloire du quelconque » (Rancière, 2000, ePub).

Sous la plume d'Arsenault, l'esthétique trash n'est pas seulement exploitée dans sa valeur la plus littérale, son extravagance est également à l'œuvre dans les « Poèmes anglais », dont la langue est crue et vulgaire, qui clôturent le recueil. Il est intéressant de relever que ces poèmes sont effectivement rédigés en anglais, comme si, pour le poète, la langue française ne permettait pas le privilège de l'extravagance langagière.

Hard Fuck

the smell of liquor

the smell of no food

the smell of puke

Hard Fuck

estrange

desperation

of being (Arsenault, 2013 [1973], p. 80)

Le bas corporel est ici aussi convoqué, mais dans un tout autre registre. Loin d'être reconnoté positivement, le bas, divulgué dans une langue particulièrement directe, ne 
ISABELLE KIROUAC MASSICOTTE, «Une esthétique trash de la marginalité. Des Crasseux d'Antonine Maillet à la collection “Poésie/Rafale ” (Perce-Neige) 》

connote que la misère («no food »), l'aliénation et le désespoir. Chez Arsenault, le traitement de l'odeur est à l'image même de l'esthétique trash, car il oscille entre transformation (l'odeur qui devient une senteur unificatrice) et disparition (l'odeur comme symptôme de la détresse d'un pan de la société).

\section{Le trash littéraire de l'école Aberdeen}

Mon intention n'étant pas ici de donner une description exhaustive du trash littéraire en Acadie, mais bien de rattacher la production récente au corpus consacré, je propose maintenant un saut dans le temps vers les années 1990, qui marquent le début du mouvement de l'école Aberdeen. On parle d'un ensemble littéraire dont la période s'échelonne environ du début de la décennie 1990 jusqu'à la moitié des années 2000 et qui compte 14 poètes, pour un total d'une quarantaine de recueils de poésie. Malgré sa grande cohérence thématique et stylistique, l'école Aberdeen n'a pas suscité beaucoup d'intérêt chez la critique, à l'exception d'Isabelle Cossette, de Manon Laparra et de Pénélope Cormier, qui ont effectué un travail de pionnières à cet égard.

À l'instar de l'esthétique trash du recueil poétique de Raymond Guy LeBlanc, celle de l'école Aberdeen se manifeste par une alternance entre silence et cri, entre invisibilité et visibilité. Ces clignotements - pour reprendre l'expression de François Paré - traduisent le rapport inégal inhérent au trash. La parole oscille entre ostentation et enfouissement, notamment dans Chroniques d'un mélodramaturge (2002) de Christian Roy, où alternent «l'ampleur de [s]on cri » et « les cauchemars muets » (p. 13), ou encore dans Ne réveillez pas l'amour avant qu'elle ne le veuille (2007) de Jean-Philippe Raîche :

En attendant que le silence monte et déborde de nous. 
Parler jusqu'à plus soif, couver le bruit du monde. (p. 25)

C'est une voix rauque, souterraine et presque surprise de sa propre élocution, « un cri lamentation nocturne/d'une gorge trop rauque» (p.46), comme l'écrit Fredric Gary Comeau dans Intouchable (1992). Le surgissement, le fait de refaire surface, liés aux enjeux de la visibilité et de l'inscription dans le domaine de la représentation, ont la valeur d'un véritable motif dans les écrits de l'école Aberdeen. Je pense entre autres aux songes que l'on sort des poubelles (p. 36) dans les vers de Marc Arseneau, auteur d'Avec l'idée de l'écho (2003) ou encore à cette strophe tirée de Ravages (1994) de Fredric Gary Comeau :

l'itinéraire du texte me rappelle les ravages de ce travail qui consiste à faire surgir le côté lumineux de la fragilité (p. 60)

Cette oscillation entre une existence souterraine et une présence lumineuse ne trouve pas de résolution dans l'écriture, porteuse de la crainte d'un «cri de terre infertile » (p. 13), comme l'écrivait Christian Brun dans Hucher parmi les bombardes (1998). Les poètes d'Aberdeen tentent d'inscrire leur voix dans le découpage du bruit et de la parole à partir d'une parole abondante et même du cri, et non pas d'un langage cru et vulgaire. Il s'agit de l'esthétique trash dans sa tendance la plus littérale, proche de la disparition et liée de près à un champ sémantique de l'enfouissement, mais aussi des déchets. Contrairement aux poèmes de LeBlanc et d'Arsenault, ceux des poètes d'Aberdeen n'opèrent pas une resignification des détritus : le potentiel transformateur du trash n'est pas actualisé. Dans les œuvres de l'école Aberdeen, le déchet est surtout lié à la pourriture et se fait le révélateur de la dégénérescence individuelle et collective. Chez Daniel Omer Leblanc et Stéphanie Morris, le déchet est interne à l'individu et demande à être transcendé. Dans Le 
ISABELLE KIROUAC MASSICOTTE, «Une esthétique trash de la marginalité. Des Crasseux d'Antonine Maillet à la collection “Poésie/Rafale " (Perce-Neige) 》

risque des rêves (2002), Morris écrit; «mon vieux sac de vidanges va resurgir/Celui de n'être que moi » (p. 19), passage qui dénote une condition à dépasser. Cela est montré un peu différemment dans Les ailes de soi (2000) de Daniel Omer Leblanc, puisque le déchet est carrément une menace à toute forme d'action : «mes déchets cérébraux bourgeonnent et attaquent/ma volonté en rémission » (p. 77). Sous la plume de Comeau, le traitement de la pourriture prend plus résolument une couleur sociale, parce qu'il est question de «cette pourriture/qui écrase toute passion» et qui «manufacture l'inhumanité » (1992, p. 24). Chez Christian Brun, tout le champ sémantique du déchet paraît mobilisé pour formuler une critique de la déchéance sociétale :

DÉCHÉANCE : milieu qui glorifie ennui et excès, convulsion de propriétés, échanges en représentation, égorgement de fissures, donation de costumes dogmatiques, pente glissante (1998, p. 89)

Cette déchéance de la société est donc multiple et concerne plusieurs formes de pouvoir, notamment le système capitaliste. Les déchets, toujours en-dehors de l'ordre dominant, se font les révélateurs du système: "where there is dirt there is system » (Douglas, 2002 [1966], p. 44). On repère chez les poètes d'Aberdeen une volonté de contester toutes les normes et pouvoirs, mais un tel projet peut difficilement aboutir. Dans leur tentative de «s'emparer de toutes les représentations de la marginalité sociale»(Cormier, 2012, p. 200), ils laissent pourtant de côté les femmes, n'étant le plus souvent dépeintes que comme l'objet du désir masculin, par exemple la «gamine délicieuse au goût sûrement sucré à croquer toute crue, toute nue, dans sa fragilité » (Roy, 2000, p. 84) dans les vers de Christian Roy. Plus encore, la subversion par un langage cru et direct rattachée au trash passe parfois par la réification de la femme, comme le montre cette strophe tirée d'Averses de Paul Bossé : 
bander

en voyant la frontière uniforme peau

de l'équipe des filles

la façon que les fesses se ferment (2004, p. 68)

Même si le trash permet de révéler l'ordre et de s'y infiltrer par un nouveau partage du sensible, il sert ici à réitérer l'ordre hétéropatriarcal. Kenneth Harrow souligne que la trashy woman, une fois dépeinte selon un regard masculin, peut être enfermée dans ce male gaze et voir son potentiel subversif bloqué. C'est ce que le chercheur appelle le «old-school trash »(Harrow, 2013, ePub), qui existe le plus souvent au détriment des femmes. La trashy woman est le symbole de l'ambiguïté de l'érotisme au féminin, qui est à la fois le lieu de l'agentivité et de la dégradation féminines. Du côté des poétesses d'Aberdeen, Stéphanie Morris écrit être une «Apprentie-anarchiste»(2002, p. 59) et son agentivité passe surtout par la sexualité : «à la suite de mes humbles jouissances et craquements de mon lit sur le rythme animal des gémissements que je peux vous soumettre pour vous rendre macho juste le temps d'une fuck d'un quickie » (p. 38). Mais la dégradation associée à la trashy woman est également à l'œuvre dans le vers « un homme qui m'annule et me crache » (Morris, 2002, p. 38); la femme trash de Morris révèle davantage l'ordre hétéropatriarcal qu'elle ne l'infiltre.

Le militantisme multiple dont parle Pénélope Cormier dans son étude de l'école Aberdeen a donc ses limites; non seulement il n'est pas intersectionnel, il est davantage latent que pratiqué; la révolution est «mijot[ée]»(2003, p. 90) chez Marc Arseneau, la révolte reste encore à «activer » $(1992$, p. 23) chez Fredric Gary Comeau et Cindy Morais « observe la manifestation qui passe » $(1999, \mathrm{p} .11)$. Le potentiel de transformation contenu dans l'engagement et le militantisme apparaît constamment court-circuité par la 
ISABELLE KIROUAC MASSICOTTE, «Une esthétique trash de la marginalité. Des Crasseux d'Antonine Maillet à la collection “Poésie/Rafale ” (Perce-Neige) »

problématique de la fin relevée par Pénélope Cormier, car l'apocalypse, emblématique de la menace de disparition intrinsèque au trash, se profile comme une présence obsédante : c'est «l'abandon nucléaire»(1992, p. 47) de Marc Arseneau, ou encore « le tourbillon de l'apocalypse » (2000, p. 9) de Christian Roy.

\section{L'esthétique trash des poètes de la relève acadienne}

Les écrits des poètes de la relève, pour la plupart publiés dans la collection «Poésie/Rafale » chez Perce-Neige, sont également traversés par une esthétique trash. Bien que cette production partage une parenté certaine avec les textes de l'école Aberdeen (les cibles y sont également multiples, j’y reviendrai), elle est davantage liée aux recueils fondateurs Cri de terre et Acadie Rock, car elle se concentre aussi sur une communauté identitaire. Je pense d'abord à l'œuvre de Sébastien Bérubé, qui est à mon avis le principal représentant du trash littéraire de la relève. Dans son premier recueil, Sous la boucane du moulin, publié en 2015, le poème «Ma terre » se lit justement comme un long « cri de terre » qui appelle à l'engagement :

J'habite une Terre violée

Que l'industrie gouverne à grands coups

De sodomie électorale

Il faut fumer à cinquante pieds de la porte

Mais la boucane du moulin Porte jusqu'aux étoiles et personne

Ne hausse le ton (p. 22)

Ces vers ne traduisent pas un champ sémantique de l'enfouissement et de la disparition; l'esthétique trash mobilisée dans cet extrait est surtout liée à un langage vulgaire et à l'utilisation de métaphores sexuelles qui visent à exprimer l'indignation face à l'inaction et au silence des citoyen.ne.s. La parenté avec l'œuvre de Raymond Guy LeBlanc est plus 
évidente dans le poème intitulé «Hymne», tiré du recueil Là où les chemins de terre finissent, car comme chez LeBlanc, les instantanés de réalités parfois accablantes n’empêchent pas d'entrevoir l'espoir :

J'ai des jeunes poètes qui font les pour et les contre

Pour se convaincre que tout n'est pas noir ou blanc

Pour se construire un hymne

Sur fond gris (Bérubé, 2017, p. 52)

Ces vers forment une sorte de commentaire méta esthétique; sans misérabilisme ni mélioration, le poète dénonce le système, mais également la léthargie de sa communauté, sevrée « au double-double/Et aux idéaux givrés/[...]/Des Tim Hortons ${ }^{\mathrm{TM}}$ aux cent pieds » (Bérubé, 2017, p. 12 et 30). Cette critique des Acadien.ne.s n'est pas sans rappeler les «cavernes honteuses de notre silence» (2012 [1972], p. 47) de LeBlanc. La plus importante particularité de Bérubé par rapport à LeBlanc est que son «cri de terre » en est un du Nord, région largement occultée en littérature acadienne.

C'est également le cas du recueil Apprendre à tomber de Jonathan Roy, qui dédie un long poème à la ruralité nordique intitulé « Voix rurale » :
une voix nord vidée
de son souffle
une voix ignorée
une voix qui se cache
une voix qui sort juste
de temps en temps (2012, p. 44)

Ont droit de parole les individus à l'existence rude, les laissés-pour-compte, les marginaux à la «voix salie », à la «voix tachée d'huile », à la «voix emphysème », à la «voix crachée » (Roy, 2012, p. 4), ainsi que les « reine[s] de cours à scrap » (Roy, 2012, p. 11), qui ne sont pas sans rappeler les Crasseux d'Antonine Maillet et les humbles dépeints dans «Acadie Expérience » de Guy Arsenault. 
ISABELLE KIROUAC MASSICOTTE, « Une esthétique trash de la marginalité. Des Crasseux d'Antonine Maillet à la collection “Poésie/Rafale ” (Perce-Neige) 》

Roy, mais plus encore Bérubé, se font les poètes des opprimés et s'attaquent aux dérives du capitalisme, à partir du Nord comme région-ressource. Bien que les jeunes poètes s'en prennent à différentes formes de pouvoirs, de normes - économiques, environnementales, médiatiques ou politiques - plusieurs poèmes laissent entrevoir une tentation du conservatisme, au sens figuré de vouloir conserver et préserver ce qui existe, notamment chez Bérubé, qu'il s'agisse «des histoires de bon vieux temps » (2017, p. 46) ou encore de la critique formulée à l'endroit de celui qui «préfère voir sa descendance/[d]ans un condom/[q]ue sur une photo de famille » $(2015$, p. 64). En ce sens, le trash des jeunes poètes de la relève est ambigu, puisque ce mouvement vers le traditionnel tranche singulièrement avec le ton parfois vulgaire et injurieux. Mais cette ambiguïté paraît néanmoins voulue par Roy, qui parle d’une « voix paradoxe » (2012, p. 47). En témoignent également la succession de vers pour le moins antithétiques comme «[j]'ai du linge de première communion/Et des chandails de Metallica étendus/Sur la même corde » $(2017$, p. 50) chez Bérubé et «une voix chrétienne/une voix païenne » (2012, p. 45) chez Roy. Peut-être ces extraits prônent-ils une cohabitation harmonieuse dans la diversité, ou encore une religiosité plus inclusive et adaptée au monde d'aujourd'hui. En somme, il semble s'agir de faire perdurer la tradition, mais quelque peu libérée de ses contraintes. C'est en ce sens que l'esthétique trash vient troubler l'ordre et provoque un nouveau partage du sensible. Le trash, c'est aussi cette absence de censure qui recense les choses potentiellement opposées de l'existence.

Il importe également de souligner l'apport, non négligeable, des poétesses de la relève au trash littéraire acadien, qui se distingue de celui mobilisé par leurs homologues masculins. Comme chez les poétesses d'Aberdeen, l'esthétique trash passe par la figure 
ambigüe de la trashy woman. Le recueil Quatre pattes Catherine de Joannie Thomas, publié en 2016, gravite autour de la vie sexuelle débridée de Catherine, dont le mode de vie nocturne est marqué par une importante consommation d'alcool et l'expérimentation de drogues. À cet égard, l'écriture se rapproche de celle des poètes d'Aberdeen, chez lesquels Isabelle Cossette et Manon Laparra ont relevé l'« utilisation littéraire de notions telles que les drogues, le sexe» (2001, p. 146). Il s'agit d'une sexualité hétérosexuelle particulièrement explicite - il n'est pas question de minorités sexuelles ici - où la femme se trouve en position active et renverse en quelque sorte le male gaze :

J'aime les corps

les fragiles

les doigts squelettiques

[...]

les mamelons durs

$[\ldots]$

les manboobs

les pancake butts

[...]

les bédaines slaques

$[\ldots]$

le poil de cul foncé (Thomas, 2016, p. 34)

La liste implique non seulement une multitude de partenaires sexuels, elle suggère aussi une absence de hiérarchie, une équivalence entre les différents éléments énumérés. L'instance narrative valorise également des traits qui ne sont pas nécessairement mis de l'avant lorsqu'il est question de masculinité, pensons à la fragilité - « les fragiles », « les doigts squelettiques »- mais aussi aux mamelons et aux manboobs. En ce sens, une certaine subversion du genre est opérée à partir d'une écriture trash. Bien que cette esthétique traverse l'ensemble de l'œuvre, les dernières pages laissent entrevoir une volonté d'entrer dans le moule, car Catherine affirme son «désir secret d'être fécondée/enfin 
ISABELLE KIROUAC MASSICOTTE, « Une esthétique trash de la marginalité. Des Crasseux d'Antonine Maillet à la collection "Poésie/Rafale " (Perce-Neige) 》

accomplie/complète » (Thomas, 2016, p. 54) et d'être dans une union aux allures très traditionnelles :

J'aurais aimé ça faire partie de ta vie

J'aurais aimé ça faire ta vaisselle sale

J'aurais aimé ça faire ton lit (Thomas, 2016, p. 55)

Comme chez Bérubé et Roy, la tentation du conservatisme est bien présente. Mais ici aussi, l'incursion de valeurs liées à une certaine préservation de l'ordre n'entraînent pas forcément le désamorçage de l'esthétique trash. La sexualité débridée de Catherine n'est pas présentée comme incompatible avec la maternité et les rôles socialement attribués aux femmes. Faute d'avoir complété le transfert vers un nouveau régime du sensible, l'écriture trash et pornographique de Thomas a néanmoins le mérite d'avoir déstabilisé et infiltré l'ordre en faisant tache sur le monde ordonné de la tradition.

Du côté de Dead end (2017), premier recueil de Monica Bolduc, l'instance narrative nous transporte elle aussi du côté des lieux nocturnes, de la consommation effrénée d'alcool et de drogues, d'une sexualité hétérosexuelle débarrassée de ses tabous et d'amours déçues, mais là s'arrête la comparaison avec Quatre pattes Catherine. La force transgressive inhérente au trash prend sa pleine mesure dans le poème qui clôt le recueil, « tu vas être belle (the twisted code of women) », qui exprime le refus d'être une femme «modèle », de correspondre à ce que la société attend de soi :

\section{Sujets à éviter :}

$[\ldots]$

Menstruations

Vomi

Marde

Pisse

Diarrhée explosive

Sexe 
Infections vaginales

Sodomie

Masturbation

Fellation

Poil de raie

Pet de plotte (Bolduc, 2017, p. 81)

Il s'agit bien de la trashy woman, «the excluded one who has no part in the spaces allotted to proper women in the dominant order »(Harrow, 2013, ePub). À partir de l'abject et de pornèmes (c'est l'expression utilisée par Marie-Anne Paveau pour désigner les termes liés à la pornographie [2014, ePub]), Bolduc formule une critique féministe. L'esthétique trash qui est à l'œuvre infiltre le partage du sensible aux niveaux visuel, sonore et olfactif. Le trash des jeunes autrices de la relève est donc surtout féministe - une dimension absente de l'écriture des hommes : Thomas présente un rapport décomplexé à la sexualité et déjoue les codes de la pornographie en présentant également divers types de corps masculins et Bolduc formule quant à elle une critique de la construction sociale des genres.

Avec cette étude, mon intention n'est pas d'imposer une nouvelle voix critique au corpus acadien, celle de l'esthétique trash, cette lecture pouvant tout à fait coexister avec d'autres. Mon objectif était surtout de montrer que l'hypothèse de Pénélope Cormier, selon laquelle la production récente peut être appréhendée à partir de la diversification des pratiques horizontales plutôt que par une rupture franche avec la génération précédente, est particulièrement féconde pour l'étude de la relève. C'est sur l'esthétique trash comme façon de «dire autrement» la marginalité sous ses différentes facettes que je me suis concentrée. Le trash m'apparaît être une esthétique privilégiée pour représenter le fait marginal en raison du rapport inégal au pouvoir qu'il sous-tend. Dans les waste studies, la 
ISABELLE KIROUAC MASSICOTTE, « Une esthétique trash de la marginalité. Des Crasseux d'Antonine Maillet à la collection "Poésie/Rafale " (Perce-Neige) 》

saleté et les déchets, toujours à l'extérieur du commun et de l'ordre dominant, sont utilisés comme métaphore pour justifier le maintien à distance des «déchets humains », ces êtres en trop qui, conscients de ce système de valeurs qui leur est imposé, s'en emparent pour l'exposer. La principale fonction du trash, véritable écriture-symptôme, est de révéler et de déstabiliser le partage du sensible, pour reprendre l'expression de Rancière.

Dans Les Crasseux, Cri de terre et Acadie Rock, l'esthétique trash s'articule autour d'une communauté identitaire, d'une acadianité. Cette acadianité s'affirme notamment par une langue vernaculaire parfois vulgaire et injurieuse, dénotant une resignification de l'injure. Toutefois, c'est chez Maillet que la dynamique de la «garbagization » est la plus prégnante, ne serait-ce qu'en raison de la division très nette entre l'En-Haut et l'En-Bas et du nom des Crasseux, rapprochés à des «déchets humains ». Ils réussissent à infiltrer l'ordre dominant et même à provoquer un certain renversement en déménageant dans l'Enhaut : c'est la révolution esthétique dont parle Rancière. Du côté de LeBlanc, le traitement du Bas est fait de façon plus implicite et passe par le champ sémantique du souterrain et de l'enfouissement, matérialisation du silence et du danger de disparition liés au trash. La question d'un nouveau partage du sensible est beaucoup plus précaire que chez Maillet, l'écriture ne trouvant pas de résolution entre le cri (une inscription stable dans le découpage du bruit et de la parole) et la terre (l'enfouissement, donc la disparition et le silence). Avec Acadie Rock, on retourne à une mise en texte du bas-corporel et des odeurs; en ce sens l'écriture d'Arsenault se rapproche davantage de celle de Maillet que de celle de LeBlanc. L'écriture trash d'Arsenault se démarque de celle de ses contemporains par la gloire du quelconque et du quotidien qu'elle représente. Mais la resignification de l'indigne, des 
odeurs, n'est pas assurée, comme en témoignent les « Poèmes anglais », traduisant un grand désespoir. Comme chez LeBlanc, l'esthétique trash oscille entre disparition et création.

Du côté des poètes d'Aberdeen, les marginalités dépeintes ne sont pas liées à l'acadianité. Malgré le fait qu'il n'est pas question de communauté identitaire dans les vers des poètes d'Aberdeen, ceux-ci partagent néanmoins une parenté avec ceux de LeBlanc, surtout dans les mouvements de l'écriture entre silence et cri, invisibilité et visibilité. Comme dans Cri de terre, la disparition se profile telle une menace, menace qui prend la forme de l'apocalypse dans les écrits de l'école Aberdeen. Bien que la métaphore du déchet soit à l'œuvre dans plusieurs poèmes, il n'y a pas de véritable resignification des détritus : ils servent plutôt à dévoiler la dégénérescence de l'individu et de la société, qu'elle soit endogène ou exogène. On s'éloigne de la jubilation créative des Crasseux et de la célébration des sans-noms chez Arsenault. Dans leur tentative de s'emparer de toutes les formes de pouvoir, les poètes d'Aberdeen échouent à s'attaquer à l'hétéropatriarcat en représentant le plus souvent les femmes dans une position sexuelle passive. La trashy woman des poétesses d'Aberdeen, à la fois figure d'agentivité et de dégradation, révèle l'ordre patriarcal plus qu'elle ne le déstabilise.

Avec les poètes de la relève associés à la collection «Poésie/Rafale », on observe un retour d'une esthétique trash pour représenter l'acadianité. Mais cette acadianité passe désormais aussi par la région, plus particulièrement le Nord. Les œuvres de Jonathan Roy et de Sébastien Bérubé font entrer dans le découpage des espaces le territoire nordique souvent dévasté et ses habitants à l'existence rude. Cette jeune relève se distingue également par une certaine ambiguïté entre changement et tradition, qui indique qu'il s'agit plus de teinter l'ordre dominant que de le renverser. Comme c'est le cas des poétesses de 
ISABELLE KIROUAC MASSICOTTE, «Une esthétique trash de la marginalité. Des Crasseux d'Antonine Maillet à la collection “Poésie/Rafale ” (Perce-Neige) 》

l'école Aberdeen, celles de la relève font figure à part. Joannie Thomas parait vouloir concilier l'image de la trashy woman à celle d'une femme plus traditionnelle; en ce sens, elle aussi teinte l'ordre. Pour sa part, Monica Bolduc critique plus directement la construction sociale des genres à partir de pornèmes et de l'abject : elle parvient à déstabiliser l'ordre. L'étude de l'esthétique trash, loin d'entraîner une lecture uniforme des œuvres acadiennes, permet au contraire de souligner toutes les ruptures, mais aussi tous les retours chez une même génération d'auteur.e.s ou d'une génération à l'autre. Le trash rend visible la précarité et les contradictions des marges, constamment en lutte pour la reconnaissance et tiraillées entre la conservation de la tradition et le changement.

\section{Bibliographie}

Arsenault, Guy (1994 [1973]), Acadie Rock, Moncton, Perce-Neige, coll. « Mémoire ». ArSenEau, Marc (1992), À l'antenne des oracles, Moncton, Perce-Neige, coll. « Poésie ». Arseneau, Marc (2003), Avec l'idée de l'écho, Moncton, Perce-Neige, coll. «Poésie ».

BEAUSOLEIL, Claude (1999), « Nouvelles voi(es/x) poétiques », Éloizes, no 28, p. 111-116.

BÉRUBÉ, Sébastien (2015), Sous la boucane du moulin, Moncton, Perce-Neige, coll. «Poésie/Rafale ».

BÉRUBÉ, Sébastien (2017), Là où les chemins de terre finissent, Moncton, Perce-Neige, coll. «Poésie/Rafale ».

Bolduc, Monica (2017), Dead end, Moncton, Perce-Neige, coll. « Poésie/Rafale ».

Bossé, Paul (2004), Averses, Moncton, Perce-Neige, coll. « Poésie ».

BOUDREAU, Raoul (2005), «Les poètes acadiens de la relève », Studies in Canadian Literature/Études en littérature canadienne, vol. 30, no 1, https://journals.lib.unb.ca/index.php/scl/article/view/15279/16366. 
Brottman, Mikita (2005), High Theory / Low Culture, Basingstoke, Palgrave Macmillan.

Brown, Cecily F. (2012), People-As-Garbage: A Metaphor We Live by, Saarbrücken, Lambert Academic Publishing.

BRUN, Christian (1998), Hucher parmi les bombardes, Moncton, Perce-Neige, coll. «Poésie ».

Comeau, Fredric Gary (1992), Intouchable, Moncton, Perce-Neige, coll. « Poésie ».

Comeau, Fredric Gary (1994), Ravages, Moncton, Perce-Neige, coll. « Poésie ».

CORMIER, Pénélope (2012), « Les jeunes poètes acadiens à l'école d'Aberdeen », dans Jacques Paquin (dir.), Nouveaux territoires de la poésie francophone au Canada : 19702000, Ottawa, Presses de l'Université d'Ottawa, coll. « Archives des lettres canadiennes », p. 179-204.

CossetTE, Isabelle et Manon Laparra (2001), «Les voix nocturnes : modes de représentations de la cité dans la poésie acadienne contemporaine », Francophonies d'Amérique, no 12, p. 145-152.

Douglas, Mary (2002 [1966]), Purity and Danger, New York, Routledge.

Doyon-Gosselin, Benoit (2016), «Je m'ennuie de Josée Yvon », Astheure, 19 octobre, https://astheure.com/2016/10/19/je-mennuie-de-josee-yvon-benoit-doyon-gosselin/

DoYON-Gosselin, Benoit (2017), «Loin d'être un cul-de-sac! », Astheure, 23 août, https://astheure.com/2017/08/23/loin-detre-un-cul-de-sac-benoit-doyon-gosselin/

HARrow, Kenneth (2013), Trash. African Cinema From Below [ePub], Bloomington, Indiana University Press.

Keller SimON, Richard (1999), Trash Culture. Popular Culture and the Great Tradition, Berkeley, University of California Press.

KEnNEDY, Greg (2007), An Onthology of Trash, New York, SUNY Press.

Leblanc, Daniel Omer (2000), Les ailes de soi, Moncton, Perce-Neige, coll. « Poésie ».

LeBlanc, Raymond Guy (2012 [1972), Cri de terre, Moncton, Perce-Neige, coll. «Poésie ».

MAILLET, Antonine (1973 [1968]), Les Crasseux, Montréal, Leméac. 
ISABELLE KIROUAC MASSICOTTE, «Une esthétique trash de la marginalité. Des Crasseux d'Antonine Maillet à la collection “Poésie/Rafale ” (Perce-Neige) 》

MCLaughlin, Mireille (2001), «Les représentations linguistiques des jeunes écrivains du Sud-Est du Nouveau-Brunswick et leur impact sur la littérature acadienne », Francophonies d'Amérique, no 12 (automne 2001), p. 133-143.

MoRAIS, Cindy (1999), Zizanie, Moncton, Perce-Neige, coll. «Poésie ».

MorRIS, Stéphanie (2002), Le risque des rêves, Moncton, Perce-Neige, coll. « Poésie ».

PARÉ, François (1992), Les littératures de l'exiguïté, Hearst, Le Nordir.

PARÉ, François (1994), Théories de la fragilité, Ottawa, Le Nordir.

PAVEAU, Marie-Anne (2014), Le discours pornographique, Paris, La Musardine.

RAîcHE, Jean-Philippe (2007), Ne réveillez pas l'amour avant qu'elle ne le veuille, Moncton, Perce-Neige, coll. « Poésie ».

RANCIÈRE, Jacques (2000), Le partage du sensible : esthétique et politique [ePub], Paris, Éditions de la Fabrique.

Robichaud, Gabriel (2018), Acadie Road, Moncton, Perce-Neige, coll. « Poésie ».

Roy, Christian (2000), Infarctus parmi les piétons, Moncton, Perce-Neige, coll. « Poésie ».

RoY, Jonathan (2012), Apprendre à tomber, Moncton, Perce-Neige, coll. « Poésie ».

Thomas, Joannie (2016), Quatre pattes Catherine, Moncton, Perce-Neige, coll. «Poésie/Rafale ».

\section{Résumé}

La production poétique acadienne des années 1990 à aujourd'hui a souvent été ignorée par la critique. Ce fut le cas de l'œuvre des poètes de l'école Aberdeen. Ces écrits se situent dans lignée d'une esthétique trash, qui se poursuit dans la production de la relève publiée dans la collection «Poésie/Rafale », chez Perce-Neige. Ces poètes poursuivent l'esthétique trash acadienne qui trouve son origine dans la pièce Les Crasseux (1973 [1968]) de Maillet ainsi que dans les recueils Cri de terre (2012 [1972]) de LeBlanc et Acadie Rock (2013 [1973]) d'Arsenault. Je souhaite démontrer que de lire la littérature acadienne sous la lorgnette du trash littéraire permet de réévaluer notre façon de catégoriser les œuvres et de rattacher la production récente au corpus acadien consacré. 


\begin{abstract}
The Acadian poetic production from the 1990s to this day has frequently been ignored by the critics. This was the case of the work of L'école Aberdeen's poets. These writings are aligned with a trash aesthetic, which continues in the young writers' production published by Perce-Neige in the "Poésie/Rafale" series. These poets pursue the Acadian trash aesthetic whose origins lie in the play Les Crasseux (1973 [1968]) by Maillet and in the poetry collections Cri de terre (2012 [1972]) by LeBlanc and Acadie Rock (2013 [1973]) by Arsenault. I intend to demonstrate that reading Acadian literature from the perspective of literary trash allows to rethink the way we categorize texts and to connect the recent production to the consecrated Acadian body of work.
\end{abstract}

\title{
Notice biobibliographique
}

Isabelle Kirouac Massicotte est professeure adjointe à l'Université de Toronto. Ses travaux portent sur le trash comme esthétique de la marginalité dans les littératures québécoise, franco-canadiennes et autochtones de langue française. Après avoir complété son doctorat en lettres françaises à l'Université d'Ottawa en 2016, elle a occupé le poste de lectrice d'échange canadienne à l'Université de Bologne, en Italie. Elle a été successivement stagiaire postdoctorale à l'Institut d'études acadiennes et au Département d'études françaises $(\mathrm{CRSH})$ de l'Université de Moncton. Elle s'intéresse à l'étude culturelle des minorités, au genre de l'horreur sociale, à l'imaginaire minier, à l'imaginaire de l'industrie et à la nordicité. Son livre Des mines littéraires. L'imaginaire minier dans les littératures de l'Abitibi et du Nord de l'Ontario, a paru en 2018 aux Éditions Prise de parole. 